\title{
Design and implementation of reduced power energy efficient binary coded decimal adder
}

\author{
N. Saravanakumar ${ }^{1}$, K. Sakthi Sudhan ${ }^{2}$, K. N. Vijeyakumar ${ }^{3}$, S. Saranya ${ }^{4}$ \\ ${ }^{1,4}$ Department of Electronics and Communication Engineering, Bannari Amman Institute of Technology, India \\ ${ }^{2}$ Department of Electronics and Communication Engineering, Adhi College of Engg and Technology Kancheepuram, India \\ ${ }^{3}$ Department of Electronics and Communication Engineering, Dr.Mahalingam College of Engineering and Technology, India
}

\begin{abstract}
Article Info
Article history:

Received Jul 25, 2019

Revised Sep 15, 2019

Accepted Sep 27, 2019

Keywords:

Constant correction

Binary to Excess Six

Conversion

Ripple carry adder

Terahertz optical asymmetric demultiplexer

ABSTRACT

This paper presents a novel architecture for low power energy binary represented decimal addition. The proposed BCD adder uses Binary to Excess Six Converter (BESC) block for constant correction to adjusts binary outputs exceeding 9 to correct decimal values and exploits the inherent advantage of reduced delay and switching, due to elimination of long carry propagation in second stage addition as in conventional design and switching OFF of the BESC block for decimal outputs less than 9. The proposed BESC-BCD adder has been designed using VHDL code and synthesized using Altera Quartus II. Experimental results demonstrates that the proposed decimal adder can lead to significant power savings and delay reduction compared to existing $\mathrm{BCD}$ adders which is realised in better power-delay product(PDP) performance. For example the PDP saving of the proposed BESC-BCD adder for a 1 digit and 2 digit addition implementations are $11.6 \%$ and $16.05 \%$ respectively, compared to the best of the designs used for comparison.
\end{abstract}

Copyright (c) 2019 Institute of Advanced Engineering and Science. All rights reserved.

\section{Corresponding Author:}

N. Saravanakumar,

Department of Electronics and Communication Engineering,

Bannari Amman Institute of Technology, India.

Email: saravanakumarnatarajan.vlsi@gmail.com

\section{INTRODUCTION AND RELATED WORK}

The binary numbering system is mostly followed in computer systems today. But in earlier days, computer systems were merely based on the decimal (base 10) numbering system rather than the binary numbering system. Decimal based computer systems are popular in systems targeted for business/commercial applications. In case of applications related to money transactions decimal arithmetic is better than binary arithmetic. Therefore, many software systems still specify the use of decimal arithmetic in their calculations. The need for efficient decimal arithmetic and its ever increasing applications in micro/minicomputers and microprocessor based equipment and appliances have been increasing.

The significance of $\mathrm{BCD}$ representation over binary representation is that it is fairly trivial to convert between the string representation of a decimal number and its $\mathrm{BCD}$ representation. This is particularly beneficial when working with fractional values, since fixed and floating point binary representations cannot exactly represent many commonly used values between zero and one (e.g., 1/10). Thus, $\mathrm{BCD}$ operations can be efficient when reading from a $\mathrm{BCD}$ device, doing a simple arithmetic operation (e.g., a single addition) and then writing the $\mathrm{BCD}$ value to some other device. Architectures and algorithms proposed to date on decimal arithmetic include: A novel $\mathrm{BCD}$ adder using redundant $\mathrm{BCD}$ addition [1]. The design involves simple conversion of a BCD number to redundant form, perform addition and reconvert the result back to $\mathrm{BCD}$ form. The extra conversion circuit used adds huge delay and increases complexity. Adder for decimal addition proposed in use speculative addition technique at the first stage [1]. The feature of the design is that it has a regular structure and the design of correction unit is independent 
of the number of input operands. In another technique, used non-speculative addition which have lesser delay [2]. Some researches use reversible logic for the design of BCD adders [3-5]. The feature of reversible logic adders is that it performs well in terms of power dissipation and logic count, however it is prone to higher delay. In a pioneer work on BCD addition, used multiplexer for the addition of correction bits [6]. The use of multiplexers for constant correction reduces delay compared to the state of art approaches.

Proposed a BCD adder using analyzer circuit for carry generation [7]. The reduced number of gates in the critical path of correction circuit reduces the total delay of the circuit. In a novel architecture for BCD addition and subtraction using three major blocks viz [8]. PG block, prefix block and the correction block. Since the correction circuit is embedded within the pre-computing blocks the design complexity is less compared to the BCD adder in [9]. proposed a novel design for decimal (BCD) adder using Terahertz Optical Asymmetric Demultiplexer (TOAD) [10].

Proposed an architecture for decimal arithmetic which can also perform binary addition [11]. The circuit has lower delay, however the design of post correction circuitry poses problems for multi-bit operands. In a pioneer work, used Carry Look Ahead(CLA) addition for the first stage being followed by carry network and correction logic in the second and third stages [12]. The architecture in demonstrate better delay reduction compared to the architecture in [11, 12], however the first stage CLA adder increases hardware complexity. A two stage correction free adder for BCD addition is proposed [13]. In stage 1, the MSB three bits of a four bit BCD number are added. In second stage the result from stage 1 is added with the LSB. Since the circuit doesn't have any extra circuit for constant correction the latency is very less compared to the previous approaches. Implemented a new architecture of BCD adder using 6-input LUTs and fast carry chains [14]. The proposed design demonstrates better critical path delay reduction however the circuit is prone to more power dissipation. Proposed a new architecture for BCD addition using flag bit generation [15]. The architecture uses flag bit computation and flag inversion logic to generate the decimal equivalent for outputs exceeding 9. The speed of the architecture is high due to use of Carry Select Adder (CSLA) [16] and CSK adder [17] for first stage addition, however it is prone to high power dissipation.

The basic objective of this work is to reduce power and latency in BCD addition by using BESC instead of 4-bit Ripple Carry Adder (RCA) as in conventional design for the correction constant addition for decimal outputs exceeding 9. The main advantage derived is realization of BESC with lesser number gates compared to a 4 bit RCA. The output of first stage adders and BESC block are passed through a multiplexer. The select signal for the multiplexer is generated from a control logic which produces 1 for sum values exceeding 9 and 0 else. The rest of the paper is organized as follows. Section 2 gives a brief description of $\mathrm{BCD}$ addition and the architecture of conventional BCD adder. Section 3 discusses about the design of proposed low power and energy efficient BESC-BCD adder. Section 4 presents the BESC logic and its detailed structure. The area and delay evaluation of the proposed and conventional decimal designs are discussed in section 5. In section 6, performance of the proposed BCD adder are discussed and compared with the previous approaches through experimental results. The implementation detail of the proposed design for a 2 digit addition is discussed in section 7. Section 8 gives a brief conclusion of the work done.

\section{OVERVIEW OF BCD ADDITION}

The addition operation of two decimal digits in BCD, together with a possible carry from previous least significant digits (assuming maximum value for input digits) viz, $9+9+1$ would result in 19 . The equivalent representation for the sum in the range 0 to 19 in binary is 0000 to 10011 and in BCD is 0000 to 1 1001(MSB 1 is carry and next four LSBs is BCD digit sum) and is shown in Table 1. It is seen from Table 1, that for the binary sum equal to or less than 1001 the corresponding BCD digit is the same. However, when the binary sum exceeds 1001, the result is invalid BCD and needs correction. The addition of $6(0110)_{2}$ to the binary sum converts it to the correct digit and a carry [3]. Based on the above methodology, the architecture of a 1digit $\mathrm{BCD}$ adder [3] is shown in Figure 1.

The input digits in binary are $\mathrm{A}\left(\mathrm{A}_{3} \mathrm{~A}_{2} \mathrm{~A}_{1} \mathrm{~A}_{0}\right)$ and $\mathrm{B}\left(\mathrm{B}_{3} \mathrm{~B}_{2} \mathrm{~B}_{1} \mathrm{~B}_{0}\right) . \mathrm{S}_{3}{ }^{\prime} \mathrm{S}_{2}$ ' $\mathrm{S}_{1}$ ' $\mathrm{S}_{0}$ ' are the sum outputs of the first stage 4 bit adder. The carry output $C_{N}$ shown in Equation (1) will be one for sum exceeding 9 or else it will be 0 . When $\mathrm{C}_{\mathrm{N}}$ is 1 , a correction constant $0110(6)$ will be added with $\mathrm{S}_{3}{ }^{\text {' }} \mathrm{S}_{2}{ }^{\prime} \mathrm{S}_{1}$ ' $\mathrm{S}_{0}$ ' by the second stage adder to produce the decimal adjusted output $S_{3} S_{2} S_{1} S_{0}$ and its realisation is shown in (2)-(5) :

$$
C_{N}=C_{\text {OUT }}+S_{3}^{\prime} S_{2}^{\prime}+S_{3}^{\prime} S_{2}^{\prime}
$$




$$
\begin{aligned}
& S_{0}=S_{0}^{\prime} \\
& S_{1}=S_{1}^{\prime} \oplus C_{N} \oplus C_{1} \\
& S_{2}=S_{2}^{\prime} \oplus C_{N} \oplus C_{2} \\
& S_{3}=S_{3}^{\prime} \oplus C_{3}
\end{aligned}
$$

Table 1. Binary and BCD equivalent representation for possible outputs of decimal addition

\begin{tabular}{ccc}
\hline Decimal output & Binary Equivalent & BCD Equivalent \\
\hline 0 & 0000 & 0000 \\
1 & 0001 & 0001 \\
& - & \\
9 & - & 1001 \\
10 & 1001 & 10000 \\
& 1010 & \\
15 & - & 10101 \\
16 & 1111 & 10110 \\
17 & 10000 & 10111 \\
18 & 10001 & 11000 \\
19 & 10010 & 11001 \\
\hline
\end{tabular}

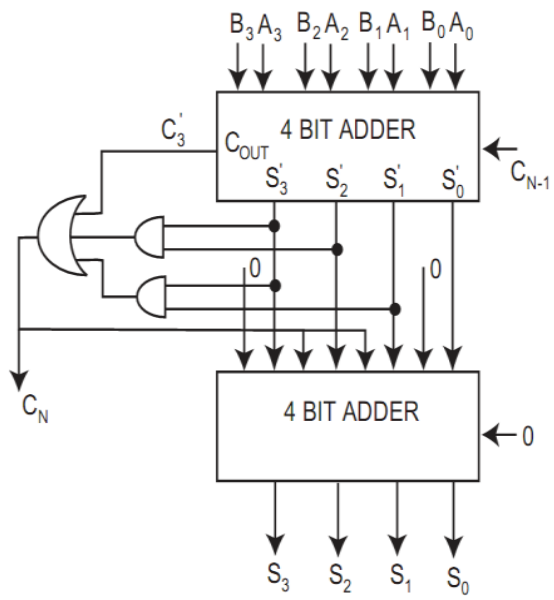

Figure 1. Block diagram of conventional BCD adder

\section{PROPOSED BESC-BCD ADDER}

The structure of the proposed BCD adder using BESC for constant correction addition is shown in Figure 2. In addition to the BESC unit, the proposed design consists of a 4 bit RCA, Carry generator and four 2:1 multiplexer (mux). The schematic of Carry generator is shown in Figure 3. When the output from first stage 4 bit RCA exceeds 9, Carry generator produces 1. The Carry generator output is used as the control/select signal for the four 2:1 multiplexer group shown as 8:4 mux in Figure 2. One input to the four 2:1 mux are the output bits of first stage adder $\left(\mathrm{S}_{3} \mathrm{~S}_{2} \mathrm{~S}_{1} \mathrm{~S}_{0}\right)$ and the other input is the BESC output $\left(\mathrm{M}_{3} \mathrm{M}_{2} \mathrm{M}_{1} \mathrm{M}_{0}\right)$. The mux selects either of the inputs based on the control signal from Carry generator $\left(\mathrm{C}_{\text {out }}\right)$ to produce the output $\left(\mathrm{O}_{3} \mathrm{O}_{2} \mathrm{O}_{1} \mathrm{O}_{0}\right)$ with $\mathrm{C}_{\text {out }}$ being the carry out bit. The main feature of the proposed decimal adder is the BESC unit which shows significant reduction in delay and switching due to elimination of carry propagation in second pair of RCA as in conventional design. 


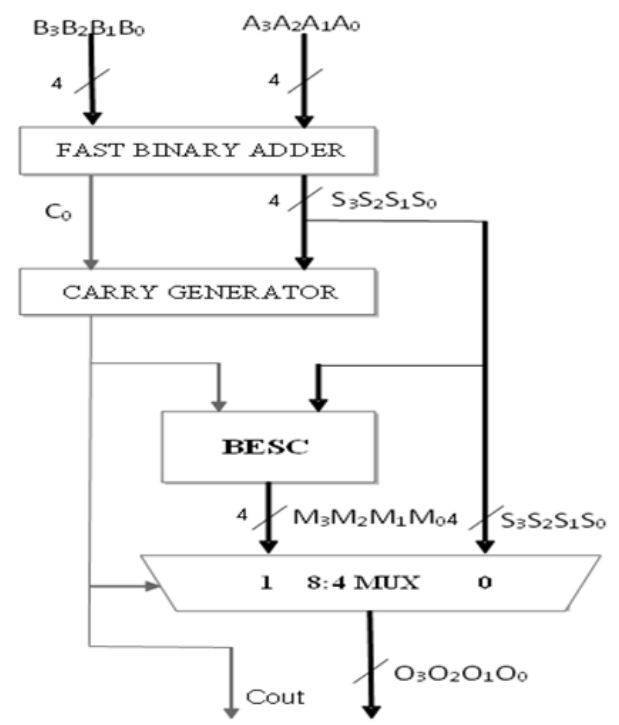

Figure 2. Block diagram of proposed BESC-BCD adder

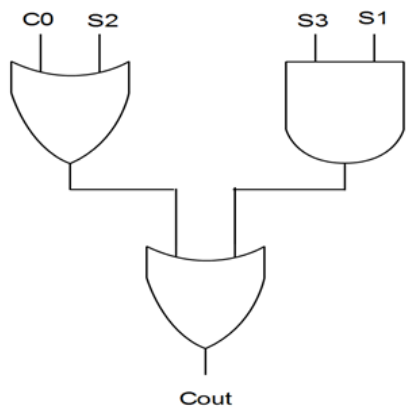

Figure 3. Schematic of carry generator

\section{BESC}

As stated in section 1, the objective of the work is to introduce BESC instead of a separate RCA for adding correction constant (0110:6) to adjust outputs exceeding 9 to correct decimal. A 4 bit BESC is used to replace 4 bit RCA for correction constant addition. The logic table which realises the binary to excess six conversions is shown in Table 2 and its schematic in Figure 4. $\mathbf{X}$-represents don't care state.

Table 2. Logic table for binary to excess 6 conversion

\begin{tabular}{lllllllll}
\hline $\mathrm{C}_{0}$ & $\mathrm{~S}_{3}$ & $\mathrm{~S}_{2}$ & $\mathrm{~S}_{1}$ & $\mathrm{~S}_{0}$ & $\mathrm{M}_{3}$ & $\mathrm{M}_{2}$ & $\mathrm{M}_{1}$ & $\mathrm{M}_{0}$ \\
\hline 0 & 0 & 0 & 0 & 0 & $\mathrm{X}$ & $\mathrm{X}$ & $\mathrm{X}$ & $\mathrm{X}$ \\
0 & 0 & 0 & 0 & 1 & $\mathrm{X}$ & $\mathrm{X}$ & $\mathrm{X}$ & $\mathrm{X}$ \\
0 & 0 & 0 & 1 & 0 & $\mathrm{X}$ & $\mathrm{X}$ & $\mathrm{X}$ & $\mathrm{X}$ \\
- & - & - & - & & $\mathrm{X}$ & $\mathrm{X}$ & $\mathrm{X}$ & $\mathrm{X}$ \\
- & - & - & - & & 0 & 0 & 0 & 0 \\
0 & 1 & 0 & 0 & 0 & 0 & 0 & 0 & 0 \\
0 & 1 & 0 & 1 & 1 & 0 & 0 & 1 & 1 \\
0 & 1 & 0 & 1 & 1 & 0 & 0 & 1 & 1 \\
0 & 1 & 1 & 0 & 0 & 0 & 1 & 0 & 0 \\
0 & 1 & 1 & 0 & 0 & 0 & 1 & 0 & 0 \\
0 & 1 & 1 & 1 & 1 & 0 & 1 & 1 & 1 \\
0 & 1 & 1 & 1 & 1 & 0 & 1 & 1 & 1 \\
1 & 0 & 0 & 0 & 0 & 1 & 0 & 0 & 0 \\
1 & 0 & 0 & 0 & 0 & 1 & 0 & 0 & 0 \\
1 & 0 & 0 & 1 & 1 & $\mathrm{X}$ & $\mathrm{X}$ & $\mathrm{X}$ & $\mathrm{X}$ \\
1 & 0 & 0 & 1 & 1 & 0 & 0 & 0 & 0 \\
\hline
\end{tabular}

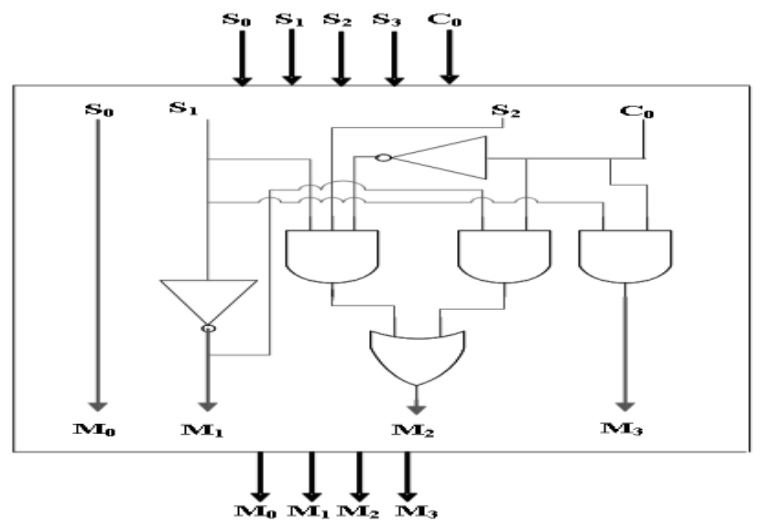

Figure 4. Schematic of BESC 


$$
\begin{aligned}
& M_{0}=S_{0} \\
& M_{1}=\overline{S_{1}} \\
& M_{2}=C_{0} \& S_{1} \& S_{2}+C_{0} \& S_{1} \\
& M_{3}=C_{0} \& S_{1}
\end{aligned}
$$

\section{AREA AND DELAY EVALUATION OF PROPOSED BESC-BCD ADDER AND CONVENTIONAL BCD ADDER}

Area and delay evaluation of the proposed and conventional designs are done based on their equivalent NAND implementation. The NAND implementation of basic elements viz., Full Adder (FA), NOT, AND and OR gates used in the proposed and conventional BCD adder designs are shown in Figure 5. We assume the delay of NAND gate to be 1 unit and area equal to 1 count. The gates in parallel between dotted lines perform parallel operation and we use only one gate delay in these cases for calculation of worst case delay of circuit/element. Based on the above approach, the delay and area of the individual elements that make up the proposed design is shown in Table 3. The area count and worst case delay of the proposed and conventional BCD adder designs are calculated as follows. The worst case delay is found by counting the number of NAND gates in the critical path and the area is evaluated by counting the total number of NAND gates that make up the circuit.

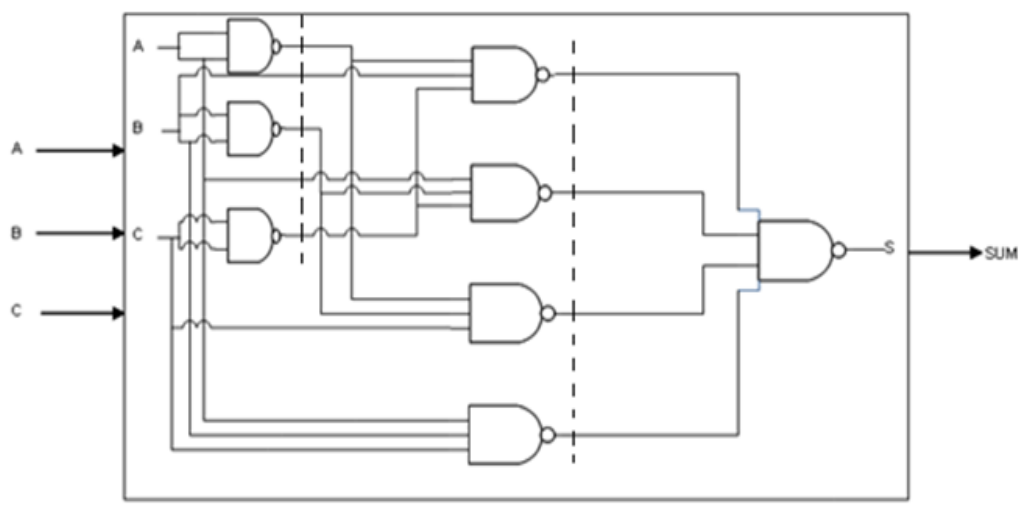

(a)

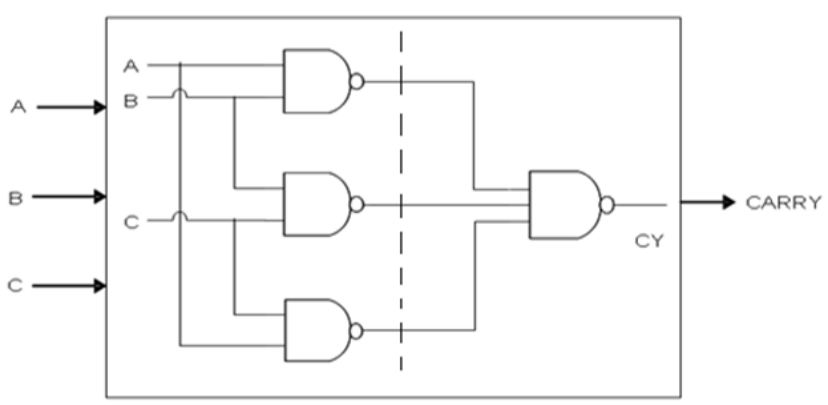

(b)

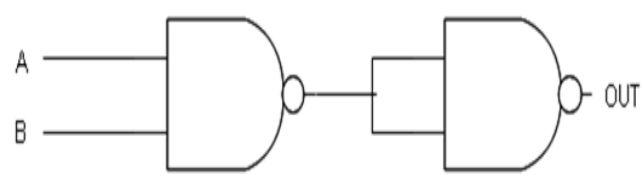

(c) 


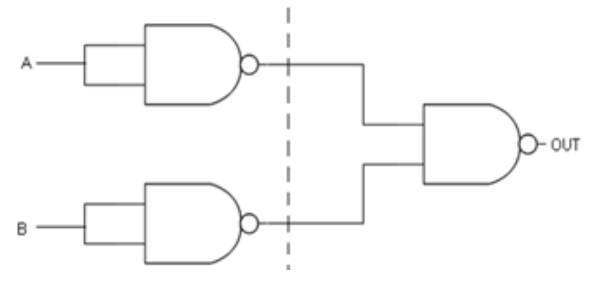

(d)

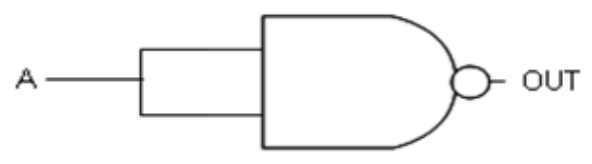

(e)

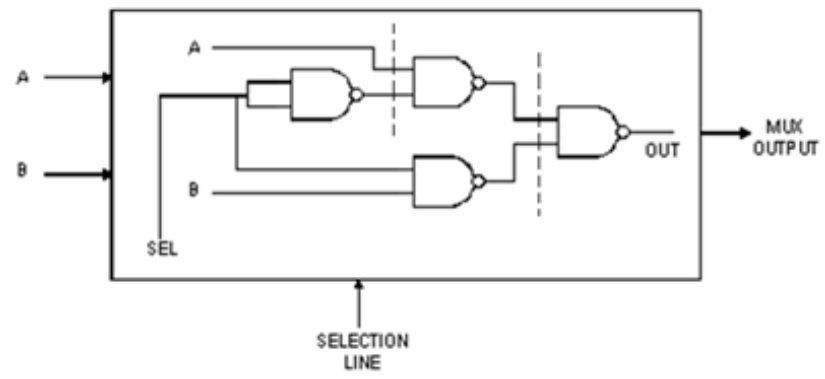

(f)

Figure 5. NAND implementation of, (a) Full adder sum, (b) Full adder carry, (c) NOT logic, (d) AND logic, (e) OR logic, (f) 2:1 Mux

Table 3. Area and delay of basic elements of BCD adder implemented using NAND logic

\begin{tabular}{ccc}
\hline Element & Area & Worst case delay \\
\hline Full adder & Sum:8 + Carry:4 12 & 3 \\
NOT & 1 & 1 \\
AND & 2 & 2 \\
OR & 3 & 2 \\
$2: 1$ Mux & 4 & 3 \\
\hline
\end{tabular}

\subsection{Area count evaluation}

The steps that lead to the area count evaluation for the BCD adder designs shown in Figure 1 and Figure 2 are as follows. From Figure 1 it can be seen that 4 FA( $\left(1^{\text {st }}\right.$ stage adder $)+2$ AND and 1 OR (Correction circuit) +4 FA ( $2^{\text {nd }}$ stage adder) make up the conventional BCD adder. Using Table 3 the NAND equivalent gate count of the decimal adder in Figure 1 is found to be $(48+4+3+8) 103$. Using a similar approach,the area count of the proposed BESC-BCD adder shown in Figure 2 is evaluated as 4 FA $\left(1^{\text {st }}\right.$ stage adder)+1 AND and 2 OR (Carry generator)+2 NOT and 3 AND and 1 OR(BESC)+16 NAND (4-2:1 mux) with corresponding NAND equivalent count being 83 . The results of the evaluation are shown in Table 4. It is seen from Table 4 that the NAND equivalent Gate count of the proposed BESC-BCD adder decreases by 20 compared to the conventional BCD adder. Worst case delay estimates (in NAND equivalent) of Conventional and proposed BESC-BCD adders as shown in Table 5.

Table 4. Area count (in NAND equivalent) of the proposed BESC-BCD adder and conventional design

\begin{tabular}{lcccc}
\hline \multicolumn{1}{c}{ Basic Blocks } & Conventional design & Area count & BESC-BCD design & Area count \\
\hline 1st stage adder & 4 FA & 48 & 4 FA & 48 \\
Correction circuit/Carry generator & 2 AND+1 OR & 7 & 1 AND +2 OR & 8 \\
2nd stage adder & 4 FA & 48 & - & - \\
BESC & - & - & 2 NOT+3 AND+1 OR & 11 \\
2:1 mux & - & - & 4 NAND & 16 \\
Total & & 103 & & 83 \\
\hline
\end{tabular}


Table 5. Worst case delay estimates (in NAND equivalent) of Conventional and proposed BESC-BCD adders

\begin{tabular}{ccccc}
\hline Basic Blocks & $\begin{array}{c}\text { Conventional } \\
\text { design }\end{array}$ & $\begin{array}{c}\text { NAND equivalent } \\
\text { delay }\end{array}$ & BESC-BCD design & $\begin{array}{c}\text { NAND equivalent } \\
\text { delay }\end{array}$ \\
\hline $\begin{array}{c}\text { 1st stage adder } \\
\text { Correction circuit/ Carry }\end{array}$ & $\begin{array}{c}\text { 4 FA } \\
\text { generator }\end{array}$ & 12 & 4 FA & 12 \\
2nd stage adder & 4 OND+1 OR & 4 & & 4 \\
BESC & 4 FA & 12 & or (1 OR+1 AND) & - \\
2:1 mux & - & - & NOT+1 AND+1 OR & 5 \\
Total & - & -28 & 3 NAND & 3 \\
\hline
\end{tabular}

\subsection{Delay evaluation}

We have evaluated the worst case delay of the proposed and conventional BCD adder designs as follows. The delay of conventional BCD adder shown in Figure 1 is 4 FA carry $\left(1^{\text {st }}\right.$ stage addition) +1 AND and 1 OR (correction circuit)+4 FA carry ( $2^{\text {nd }}$ stage addition). Based on the consideration of the delay values for individual elements (in NAND equivalent implementation) shown in Table 3, the worst case delay of conventional BCD adder is estimated to be $(12+4+12) 28$. Using a similar approach the delay of proposed BESC-BCD adder shown in Figure 2 is 4 FA carry $\left(1^{\text {st }}\right.$ stage addition $)+1$ AND/1 OR and 1 OR (Carry generator)+1 NOT and 1 AND and 1 OR(BESC)+3 NAND (2:1 Mux) with the corresponding NAND equivalent delay to be $(12+4+5+3) 24$. The results of the evaluation are shown in Table 5 . It is seen from Table 5 that the delay of the proposed BESC-BCD adder decreases by 4 units compared to the conventional BCD adder [18].

\section{EXPERIMENTAL RESULTS}

The proposed BESC-BCD adder is designed using VHDL and synthesized using Altera Quartus II with EP2C35F672C6 device. Conventional BCD adder [19], Correction free BCD adder [13], Carry skip BCD adder [20] and Flagged BCD adder [15] are used for comparison. The designs used for comparison are also described using VHDL and synthesized using Altera Quartus II with the same device. The area, delay and total power dissipation results of proposed and previous designs are shown in Table 6. It is seen from Table 6, that the proposed BESC-BCD adder has lower power dissipation and reduced delay.

Table 6. Comparison of area, delay, power dissipation and PDP of proposed BESC-BCD adder and state-of

\begin{tabular}{|c|c|c|c|c|c|}
\hline $\begin{array}{c}\text { Parameters BCD } \\
\text { Adders }\end{array}$ & $\begin{array}{l}\text { Area (Number } \\
\text { of LEs) }\end{array}$ & $\begin{array}{c}\text { Delay } \\
\text { (ns) }\end{array}$ & $\begin{array}{c}\text { Static power dissipation } \\
(\mathrm{mW})\end{array}$ & $\begin{array}{c}\text { Total power } \\
\text { dissipation }(\mathrm{mW})\end{array}$ & $\begin{array}{l}\text { Energy dissipation- } \\
\text { PDP (mW-ns) }\end{array}$ \\
\hline Conventional design[3] & 11 & 15.230 & 80.04 & 144.06 & 2194.03 \\
\hline Correction free[16] & 29 & 13.326 & 80.05 & 147.02 & 1959.19 \\
\hline Carry skip[9] & 15 & 17.100 & 80.02 & 138.36 & 2365.96 \\
\hline $\begin{array}{l}\text { Flagged BCD-Using } \\
\text { CSLA[20] }\end{array}$ & 13 & 12.303 & 80.04 & 146.43 & 1800.7 \\
\hline $\begin{array}{l}\text { Flagged BCD -Using } \\
\text { CSK[20] }\end{array}$ & 9 & 12.984 & 80.04 & 146.31 & 1899.1 \\
\hline $\begin{array}{l}\text { Proposed-BESC BCD } \\
\text { (RCA in } 1^{\text {st }} \text { stage) }\end{array}$ & 11 & 11.815 & 80.01 & 134.75 & 1592.1 \\
\hline $\begin{array}{l}\text { Proposed-BESC BCD } \\
\text { (CSLA in } 1^{\text {st }} \text { stage) }\end{array}$ & 13 & 11.657 & 80.01 & 137.32 & 1600.73 \\
\hline $\begin{array}{l}\text { Proposed-BESC BCD } \\
\left.\text { (CSK in } 1^{\text {st }} \text { stage }\right)\end{array}$ & 11 & 11.815 & 80.01 & 134.53 & 1589.47 \\
\hline
\end{tabular}

Compared to all other architectures used for comparison, thanks to the BESC unit which realises constant correction addition with minimal switching and has fewer gates in the critical path. The energy dissipation represented as product of power and delay (PDP) of the Proposed BESC-BCD adder is 11.6\% lower compared to the best of the previous designs used for comparison. The area of the proposed BCD adder represented in Logic Element (LE) count is low compared to Correction free [13] and Carry skip [20] $\mathrm{BCD}$ designs. However the proposed BESC-BCD adder show high LE count compared to the Flagged BCD design (with CSK in the first stage addition) [15]. 


\section{IMPLEMENTATION OF PROPOSED BCD-BESC ADDER FOR TWO DIGIT ADDITION}

To determine the functionality of the proposed BCD adder an implementation in 2 digit addition is done and is shown in Figure 6. The carry out of the first digit adder $\left(\mathrm{C}_{\text {out }}\right)$ is used as the carry input for 4 bit RCA in second digit addition. The performance parameters of 2 digit adder implemented with proposed BESC-BCD adder and other approaches are shown in Table 7. From the Altera Quartus II results, it is seen that 2 digit addition using proposed BESC-BCD adder designs exhibit lower delay and reduced total power dissipation compared to all other architectures used for comparison. The reduction in delay is due to realisation of second stage constant correction with $1 \mathrm{NOT}+1$ AND+1OR which eliminates the long carry propagation path as in Conventional BCD adder[3], Correction free [13], Carry skip [20] and Flagged BCD adder [15] designs. The better delay and power dissipation performance of the proposed BESC-BCD design demonstrates better PDP product of the proposed design compared to all other architectures used for comparison.

Table 7. Comparison of area, delay and power dissipation and their products of proposed BESC-BCD adder

\begin{tabular}{|c|c|c|c|c|c|}
\hline $\begin{array}{c}\text { Parameters BCD } \\
\text { Adders }\end{array}$ & $\begin{array}{c}\text { Area } \\
\text { (Number of LEs) }\end{array}$ & $\begin{array}{l}\text { Static power } \\
\text { dissipation } \\
(\mathrm{mW})\end{array}$ & $\begin{array}{l}\text { Static power } \\
\text { dissipation } \\
(\mathrm{mW})\end{array}$ & $\begin{array}{l}\text { Total power } \\
\text { dissipation } \\
(\mathrm{mW})\end{array}$ & $\begin{array}{l}\text { Energy dissipation } \\
\text { (PDP) (mW-ns) }\end{array}$ \\
\hline Conventional[3] & 24 & 89.05 & 89.05 & 172 & 3167.38 \\
\hline Correction free [16] & 58 & 89.34 & 89.34 & 173.06 & 2825.38 \\
\hline Carry skip[9] & 32 & 91.56 & 91.56 & 174.27 & 3680.58 \\
\hline $\begin{array}{c}\text { Flagged BCD-Using } \\
\text { CSLA[20] }\end{array}$ & 25 & 80.14 & 80.14 & 175.39 & 2744.85 \\
\hline $\begin{array}{c}\text { Flagged BCD -Using } \\
\text { CSK[20] }\end{array}$ & 21 & 86.83 & 86.83 & 169.22 & 2791.11 \\
\hline $\begin{array}{l}\text { Proposed-BESC BCD } \\
\text { (RCA in } 1^{\text {st }} \text { stage) }\end{array}$ & 21 & 80.09 & 80.09 & 158.75 & 2304.26 \\
\hline $\begin{array}{l}\text { Proposed-BESC BCD } \\
\text { (CSLA in } 1^{\text {st }} \text { stage) }\end{array}$ & 26 & 80.10 & 80.10 & 163.66 & 2323.32 \\
\hline
\end{tabular}

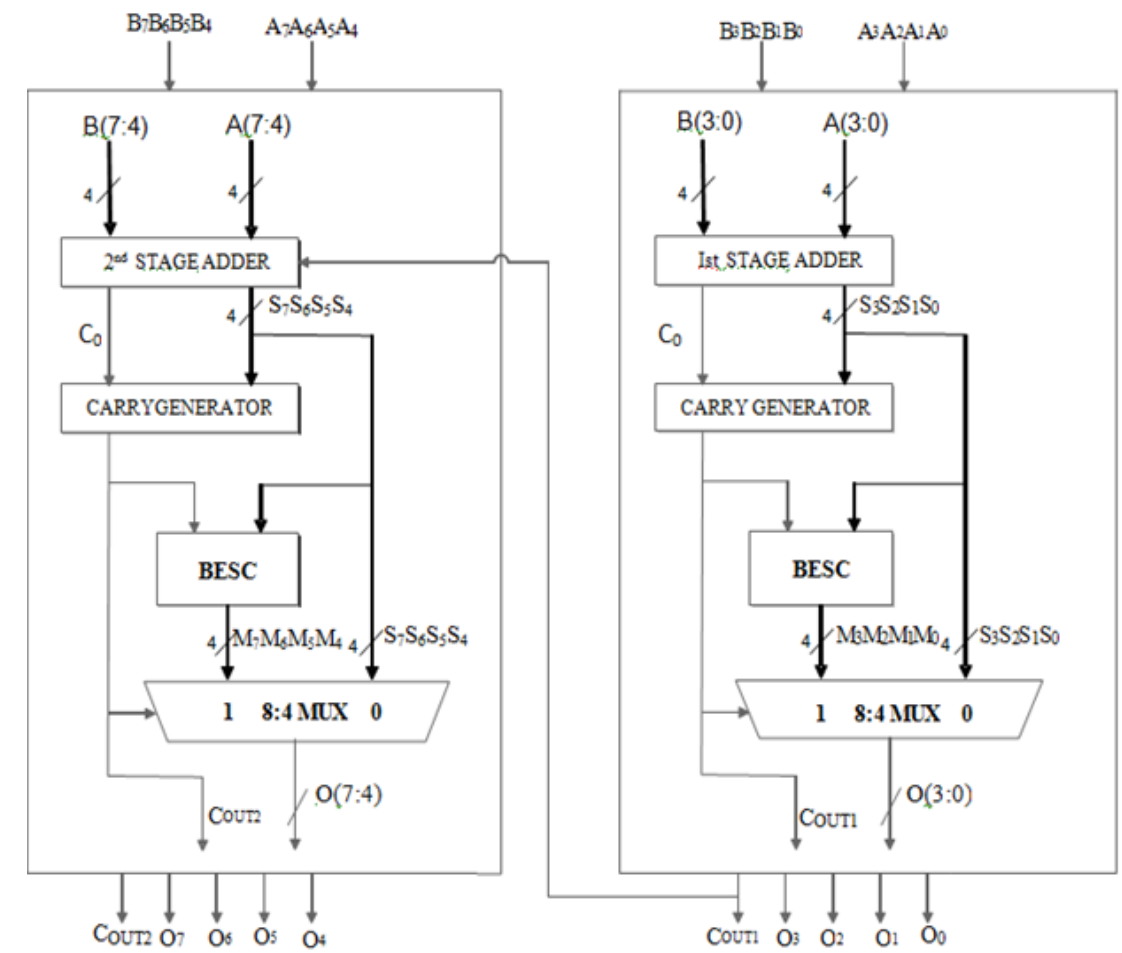

Figure 6. Block diagram of 2 digit adder implemented with proposed BESC-BCD adder 


\section{CONCLUSION}

We have proposed a new adder for decimal addition using the concept of binary-excess six conversions for constant correction, to adjust outputs exceeding 9 to equivalent decimal. An extensive evaluation of area and worst-case delay of the proposed decimal adder with conventional design, fair better results which proves the novelty of the proposed methodology. Extensive comparison using synthesis results shows that the proposed BESC-BCD adder outperformed all other previous designs in terms of power dissipation and delay performances. The potential benefits of reduced delay and power dissipation of our proposed BESC-BCD adder can be realized in fair PDP saving performance. This suggests the suitability of our design for portable VLSI implementation. Moreover, the proposed methodology for decimal addition can be easily extended to multi digits.

\section{REFERENCES}

[1] Behrooz Shirazi, David Y.Y.Yun, and Chang N.Zhang,"VLSI Designs for Redundant Binary-Coded Decimal Addition", Proc. of $7^{\text {th }}$ IEEE International Phoenix Conference on Computers and Communications, Scottsdale, AZ, pp.52-56, March 1988

[2] Robert D. Kenney and Michael J. Schulte, "High-Speed Multi-operand Decimal Adders", IEEE Transactions On Computers, Vol.54, No.8, pp.953-963, August 2005.

[3] Hafiz Mohammed Hasan Babu and Ahsan Raja Chowdhury, "Design of a Reversible Binary Coded Decimal Adder by Using Reversible 4-bit Parallel Adder", Proc. of $18^{\text {th }}$ IEEE International Conference on VLSI design (VLSID), 2005.

[4] Himanshu Thapliyal, Saurabh Kotiyal and M.B Srinivas, "Novel BCD Adders and their Reversible Logic Implementation for IEEE 754r Format", Proc. of the 19th International Conference on VLSI Design (VLSID’06) 2006.

[5] Himanshu Thapliyal and Nagarajan Ranganathan, "A New Reversible Design of BCD Adder", in Proc. of Design, Automation and Test in Europe Conference \& Exhibition (DATE), pp. 1-4, 2011

[6] Sreehari Veeramachaneni, M.Kirthi Krishna, Lingamneni Avinash, Sreekanth Reddy M.B.Srinivas, "Novel, HighSpeed 16-Digit BCD Adders Conforming to IEEE 754r Format" IEEE Computer Society Annual Symposium on VLSI (ISVLSI'07), 2007.

[7] Alp Arslan Bayrakci and Ahmet Akkas, "Reduced Delay BCD Adder", IEEE International Conference on Application Specific Systems, Architectures and Processors(ASAP), Montreal, Que., pp.266-271, 2007.

[8] Anshul Singh, Aman Gupta, Sreehari Veeramachaneni and M.B. Srinivas, "A High Performance Unified BCD and Binary Adder/Subtractor”, IEEE Computer Society Annual Symposium on VLSI, pp.211-216 2009.

[9] Sreehari Veeramachaneni.M, Kirthi Krishna.V, Prateek G.S, Subroto.S, Bharat, M.B.Srinivas, "A Novel Carry- Look Ahead Approach to a Unified BCD and Binary Adder/Subtractor", 21st International Conference on VLSI Design 2008, pages 547-552, January 2008.

[10] Gayen D.K, Bhattacharyya A, Pal R.K, Roy, J.N "All optical binary coded decimal adder" in Proc. of $4^{\text {th }}$ International conference on Computers and Devices for communication, Kolkata, pp.1-4, 2009.

[11] Chetan Kumar V, Sai Phaneendra P, Syed Ershad Ahmed, Sreehari Veeramachaneni, N, M.B Srinivas,"A Unified Architecture for BCD and Binary Adder/Subtractor", in Proc. of 14th Euromicro Conference on Digital System Design pp.426-429 2011.

[12] SundaresanC, Chaitanya CVS, PR Venkateswaran, Somashekara Bhat and Mohan Kumar J, "Modified Reduced Delay BCD Adder", Proc. of IEEE4th International Conference on Biomedical Engineering and Informatics (BMEI) 2011, pages 2148-2151.

[13] Osama Al-Khaleel, Mohammad Al-Khaleel, Zakaria Al-Qudah,Christos A. Papachristou and Khaldoon Mhaidat, and Francis G. Wolff, "Fast Binary/Decimal Adder / Subtractor with a Novel Correction-Free BCD Addition" Proc. of $18^{\text {th }}$ IEEE International Conference on Electronics, Circuits and Systems(ICECS), Beirut, pp.455-459 2011.

[14] Shuli Gao, Al-khalili,D, Chabini N, "An improved BCD adder using 6-LUT FPGAs" Proc. of 10th IEEE International New circuits and systems conference(NEWCAS), Montreal, QC, pp.13-16, 2012.

[15] Vijeyakumar, K.N, Sumathy, V. Dinesh babu, A. Elango S. and Saravanakumar, S. "FPGA implementation of low power Hardware efficient Flagged Binary Coded Decimal Adder", International Journal of Computer Applications, Vol 46, No.14, pp.41-45, May 2012.

[16] B. Ramkumar and Harish M Kittur, "Low-Power and Area-Efficient Carry Select Adder", IEEE transactions on Very Large Scale Integration (VLSI) systems, Vol. 20, No. 2, pp.371-375, February 2012

[17] Min Cha Earl.E.Swartzlander, Jr. "Modified carry-skip adder for reducing first block delay", Proc. of 43rd Midwest symposium on circuits and systems, August 2000.

[18] Vibhuti Dave, Erdal Oruklu and Jafar Saniie, "Constant addition with flagged binary adder arhitectures", Integration the VLSI journal, Vol.43, pp. 258-267, 2010.

[19] Moris Mano - Digital design 3rd edition Prentice Hall Puclication 2001.

[20] Ashis Kumer Biswas, Md. Mahmudul Hasan, Moshaddek Hasan, Ahsan Raja Chowdhury and Hafi Md. Hasan Babu, "A Novel Approach to Design BCD Adder and carry Skip BCD Adder", 21st International Conference on VLSI Design pp. 566-571 2008. 